\title{
Fluid Leakage in Static Rubber Seals
}

\author{
B. N. J. Persson ${ }^{1,2}$
}

Received: 1 December 2021 / Accepted: 1 February 2022 / Published online: 17 February 2022

(c) The Author(s) 2022

\begin{abstract}
Interfacial surface roughness can result in fluid leakage of seals, and in the design of seals it is standard to give an upper limit for the surface root-mean-square (rms) roughness amplitude $h_{\mathrm{rms}}$. However, $h_{\mathrm{rms}}$ is determined mainly by the long-wavelength roughness, which is (nearly) irrelevant for the sealing. I discuss the parameters which determine the leakage of seals, and present results for static rubber seals with circular cross-section (like rubber O-rings). I also study the influence of the fluid pressure on the interfacial surface separation and the leakrate.
\end{abstract}

\section{Graphical Abstract}

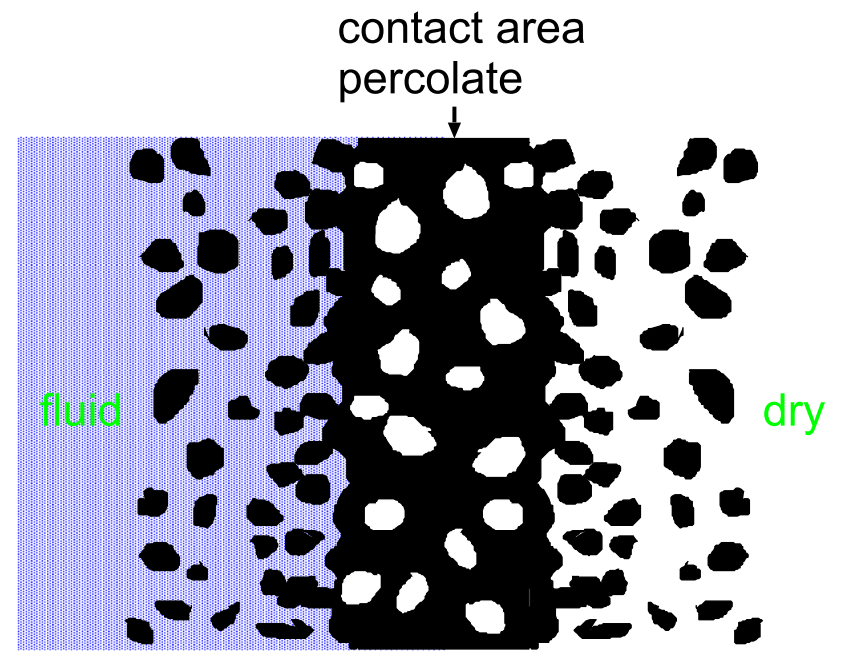

Keywords Rubber seals $\cdot$ Rubber O-rings $\cdot$ Fluid leakage $\cdot$ Percolation $\cdot$ Contact area $\cdot$ Elasticity

\section{Introduction}

Leakage of fluids through interfaces formed between rough surfaces squeezed together is a topic of great practical importance and its neglect can lead to huge economic losses and

\section{B. N. J. Persson}

b.persson@fz-juelich.de

1 Peter Grünberg Institute (PGI-1), Forschungszentrum Jülich, 52425 Jülich, Germany

2 MultiscaleConsulting, Wolfshovener str. 2, 52428 Jülich, Germany environmental problems. Here we are interested in rubber seals with circular (or half circular) cross-section squeezed against a counter surface, see Fig. 1. Examples of such seals are rubber O-rings or the ribs on the rubber stoppers in syringes. For rubber seals we can assume purely elastic deformations (no plastic flow) and if the surface roughness is not too big the nominal (or macroscopic) contact pressure will be Hertz-like (parabolic) [1] but the microscopic pressure profile can be very complex due to surface roughness.

In the design of seals limits on the surface roughness is usually specified. In the literature the rms roughness amplitude, $h_{\mathrm{rms}}$, is used to characterize the roughness and typically 


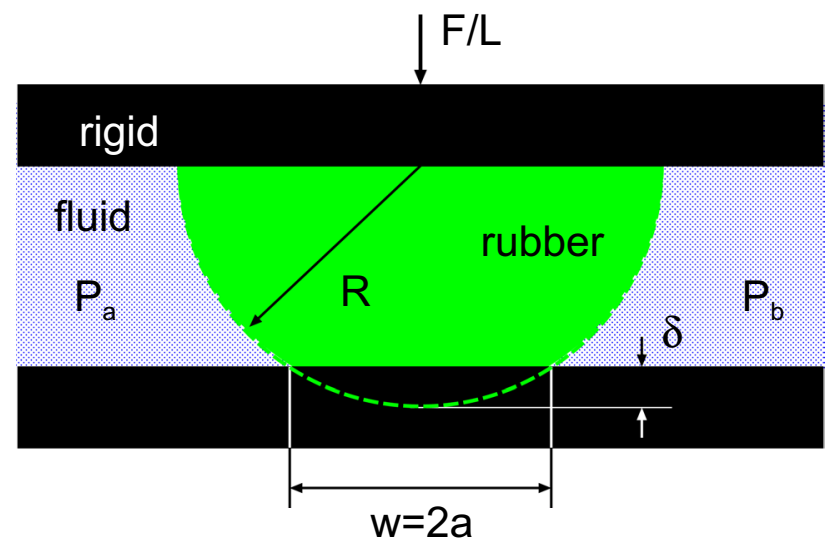

Fig. 1 Half rubber O-ring between two solid walls. The O-ring act with a force per unit length $F / L$ on the solid walls. The compression $\delta / R$ is determined by the penetration $\delta$ and the radius $R$ of the O-ring cross-section

for static seals it should be below $\approx 1 \mu \mathrm{m}$ (see, e.g., $[2,3]$ ). However, $h_{\mathrm{rms}}$ is determined mainly by the long-wavelength roughness [4] which is (nearly) irrelevant for the sealing.

For complete sealing (no fluid leakage) the area of real contact must percolate. For surfaces with random roughness this occur when the relative contact area [5] $A / A_{0} \approx 0.42$. The area of real contact $A$ is determined by the rubber viscoelastic modulus, and by the surface rms-slope (denoted $\xi$ ), which depends on the roughness on all length scales and in particular on the shortest wavelength roughness.

Rubber seals are usually confined between the surfaces of two (nearly) rigid solids at a fixed compression $\delta / R$ (see Fig. 1). I will show that if $\delta / R$ is fixed and if the fluid pressure is negligible compared to the rubber-countersurface pressure, then the fluid leakage rate does not depend on the elastic modulus of the rubber seal. This fundamental result differs from what is stated in the literature. In this paper I will also consider the dependency of the leakage rate as the fluid pressure approach the rubber-countersurface contact pressure, resulting in "lift-off" and a strong increase in the fluid leakrate [6].

\section{Surface Roughness Power Spectra and the rms Surface Slope}

All surfaces of solids have surface roughness and many surfaces exhibit self-affine fractal behavior [7]. This implies that if a surface area is magnified new (shorter wavelength) roughness is observed which appears very similar to the roughness observed at smaller magnification, assuming that the vertical coordinate is scaled with an appropriate factor. The roughness profile $z=h(\mathbf{x})$ of a surface can be written as a sum of plane waves $\exp (i \mathbf{q} \cdot \mathbf{x})$ with different

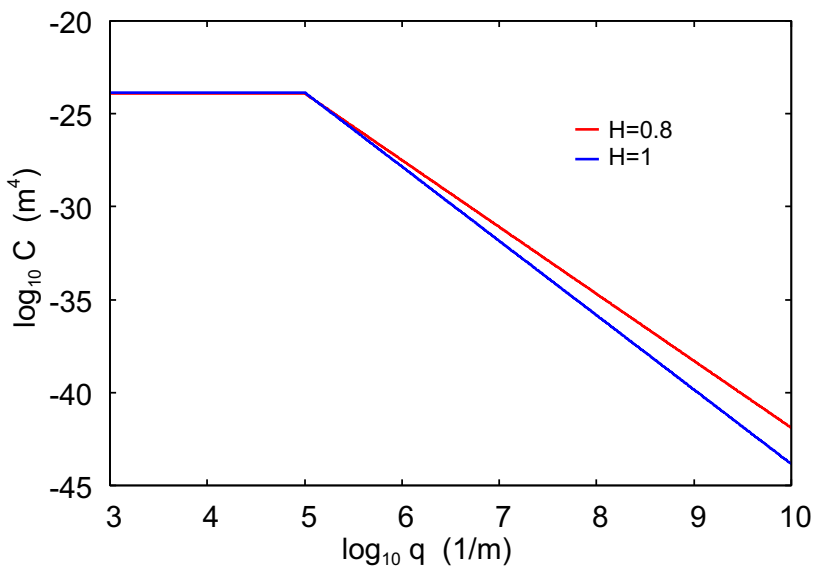

Fig. 2 The power spectra of two surfaces with the Hurst exponent $H=1$ (blue) and $H=0.8$ (red), as a function of the wavenumber. Both surfaces have the root-mean-square (rms) roughness amplitude $h_{\mathrm{rms}}=0.3 \mu \mathrm{m}$ but the rms-slope differs: It is $\xi=0.103$ and 0.445 for the $H=1$ and $H=0.8$ surfaces, respectively. Both surfaces have the large wavenumber cut-off $q_{1}=10^{10} \mathrm{~m}^{-1}$, the roll-off wavenumber $q_{\mathrm{r}}=10^{5} \mathrm{~m}^{-1}$ and the small wavenumber cut-off $q_{0}=10^{3} \mathrm{~m}^{-1}$ (Color figure online)

wave vectors $\mathbf{q}$. The wavenumber $q=|\mathbf{q}|=2 \pi / \lambda$, where $\lambda$ is the wavelength of one roughness component. A self-affine fractal surface has a two-dimensional (2D) power spectrum $C(\mathbf{q}) \sim q^{-2(1+H)}$ (where $H$ is the Hurst exponent related to the fractal dimension $D_{\mathrm{f}}=3-H$ ), which is a is a strait line with the slope $-2(1+H)$ when plotted on a log-log scale. Most solids have surface roughness with the Hurst exponent $0.7<H<1$ (see Ref. [8]).

The most important information about the surface topography of a rough surface is the surface roughness power spectrum. The power spectrum of a surface $z=h(x, y)$ is given by $[4,8,9]$

$C(\mathbf{q})=\frac{1}{(2 \pi)^{2}} \int d^{2} x\langle h(\mathbf{x}) h(\mathbf{0})\rangle e^{i \mathbf{q} \cdot \mathbf{x}}$

where $\langle.$.$\rangle stands for ensemble averaging. In what follows$ we will assume isotropic roughness so that the power spectrum $C(q)$ depends only on the magnitude $q=|\mathbf{q}|$ of the wavevector.

Contact mechanics theory [10] shows that the contact between two solids with different surface roughness $h_{1}(\mathbf{x})$ and $h_{2}(\mathbf{x})$, and different elastic properties (Young's modulus $E_{1}$ and $E_{2}$, and Poisson ratio $v_{1}$ and $v_{2}$ ) can be mapped on a problem of the contact between an elastic half space (with the effective modulus $E^{*}$ and Poisson ratio $v=0$ ) with a flat surface, and a rigid solid with the combined surface roughness $h(\mathbf{x})=h_{1}(\mathbf{x})+h_{2}(\mathbf{x})$. If the surface roughness on the two surfaces are uncorrelated then the surface roughness power spectrum of the rigid surface 
$C(q)=C_{1}(q)+C_{2}(q)$,

where $C_{1}(q)$ and $C_{2}(q)$ are the power spectra of the original surfaces. The effective modulus of the elastic solid is determined by

$\frac{1}{E^{*}}=\frac{1-v_{1}^{2}}{E_{1}}+\frac{1-v_{2}^{2}}{E_{2}}$

For randomly rough surfaces, all the (ensemble averaged) information about the surface is contained in the power spectrum $C(q)$. For this reason the only information about the surface roughness which enter in contact mechanics theories (with or without adhesion) is the function $C(q)$. Thus, the (ensemble averaged) area of real contact, the interfacial stress distribution and the distribution of interfacial separations, are all determined by $C(q)$ (see Ref. [11-13]).

Note that moments of the power spectrum determines standard quantities which are output of most stylus instruments and often quoted. Thus, for example, the mean-square (ms) roughness amplitude is given by $[4,8,9]$

$\left\langle h^{2}\right\rangle=2 \pi \int_{q_{0}}^{q_{1}} d q q C(q)$.

and the ms-slope

$\left\langle(\nabla h)^{2}\right\rangle=\xi^{2}=2 \pi \int_{q_{0}}^{q_{1}} d q q^{3} C(q)$.

The rms-slope is denoted with $\xi$. Here we have assumed that the surface roughness has isotropic statistical properties, but

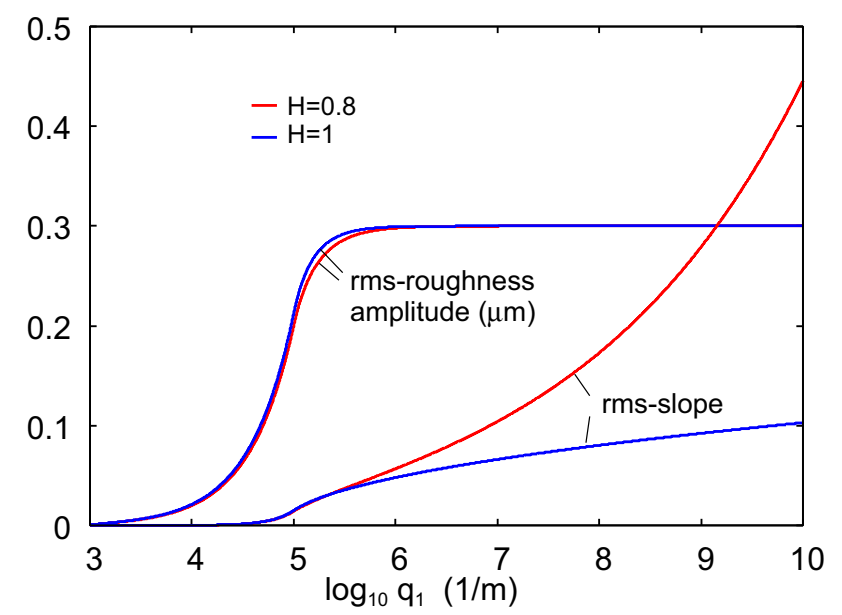

Fig. 3 The rms roughness amplitude (in $\mu m$ ) and the rms-slope of two surfaces with the Hurst exponent $H=1$ (blue) and $H=0.8$ (red), as a function of the cut-off wavenumber. When including all the roughness both surfaces have the root-mean-square (rms) roughness amplitude $h_{\mathrm{rms}}=0.3 \mu \mathrm{m}$ but the rms-slope differs: It is $\xi=0.103$ and 0.445 for the $H=1$ and $H=0.8$ surfaces, respectively (Color figure online)
(4) and (5) are also valid for anisotropic roughness in $C(q)$ is considered as the angular average (in $\mathbf{q}$-space) of $C(\mathbf{q})$.

Surfaces used for rubber seals have typically the rms roughness between $0.1 \mu \mathrm{m}<h_{\mathrm{rms}}<1 \mu \mathrm{m}$, and the Hurst exponent $0.7<H<1$. Figure 2 shows the power spectra of two surfaces with the Hurst exponent $H=1$ (blue) and $H=0.8$ (red), as a function of the wavenumber. Both surfaces have the root-mean-square (rms) roughness amplitude $h_{\mathrm{rms}}=0.3 \mu \mathrm{m}$ but the rms-slope differs: It is $\xi=0.103$ and 0.445 for the $H=1$ and $H=0.8$ surfaces, respectively. Both surfaces have the large wavenumber cut-off $q_{1}=10^{10} \mathrm{~m}^{-1}$, the roll-off wavenumber $q_{\mathrm{r}}=10^{5} \mathrm{~m}^{-1}$ and the small wavenumber cut-off $q_{0}=10^{3} \mathrm{~m}^{-1}$. Note that the power spectra differs a lot for large wavenumber, but the rms roughness is determined mainly by the long-wavelength roughness which is very similar in both cases. The rms-slope depends on the large wavenumber roughness which is much larger for the $H=0.8$ surface then for the $H=1$ surface. This fact is illustrated in detail in Fig. 3.

Figure 3 shows the cumulative rms roughness amplitude (in $\mu \mathrm{m}$ ) and the rms-slope of the two surfaces $H=1$ (blue) and $H=0.8$ (red), as a function of the large wavenumber cut-off. The results are obtained from (4) and (5) where we have increased the large wavenumber cut-off $q_{1}$ from $q_{0}=10^{3} \mathrm{~m}^{-1}$ to its final value $10^{10} \mathrm{~m}^{-1}$. Note that the rms roughness in both cases is determined by the small wavenumber region of the power spectra, while the rmsslope depends on the roughness on all length scales, and for $H=0.8$ the largest wavenumber (short wavelength) region gives the biggest contribution.

Figure 3 shows that the rms roughness is irrelevant for the leakage of seals since the contact area is determined by the rms-slope $\xi$, which is much bigger for the $H=0.8$ surface

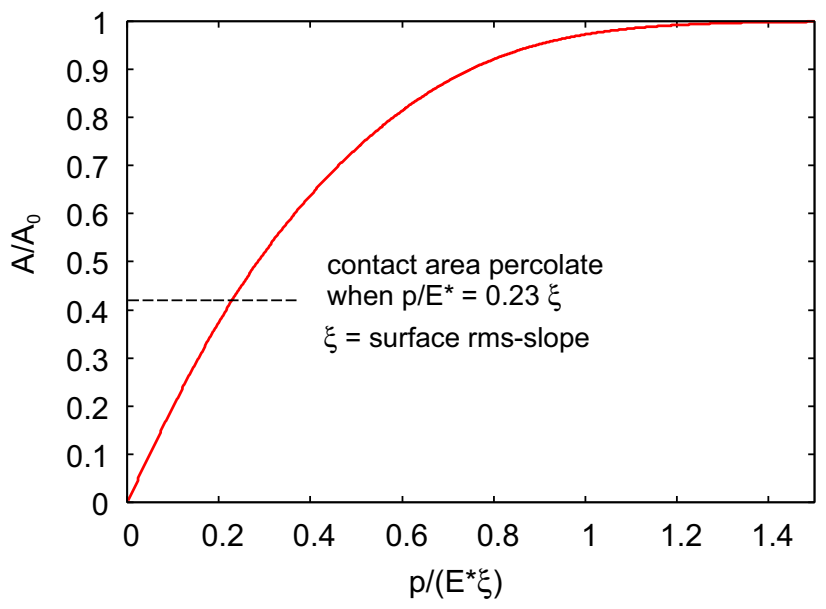

Fig. 4 The calculated area of real contact as a function of the nominal contact pressure divided by $E^{*}=E /\left(1-v^{2}\right)$ and the rms-slope $\xi$ of the rough surface. The contact area percolate when $p \approx 0.23 \xi E^{*}$ 


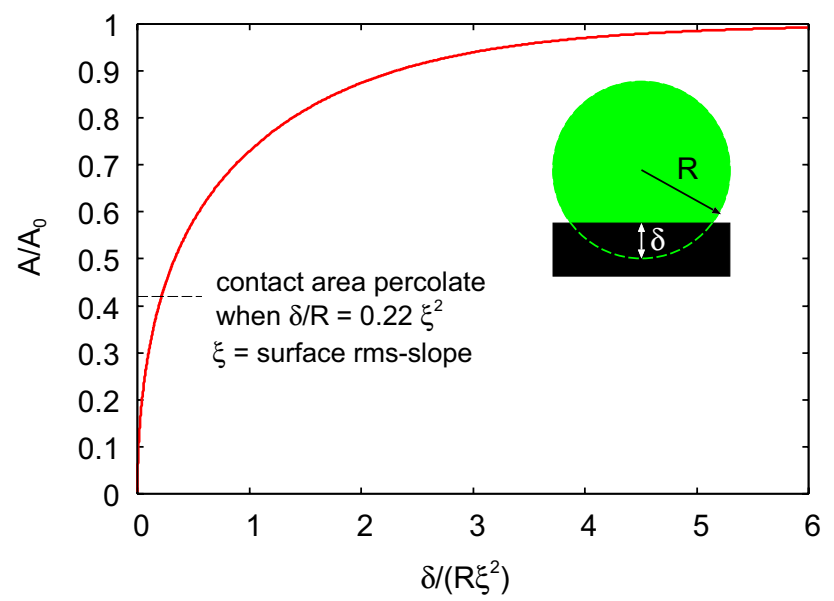

Fig. 5 The calculated area of real contact as a function of the penetration $\delta$ divided by the radius $R$ of the rubber cylinder and the square of the rms-slope $\xi$. The contact area percolate when the compression $\delta / R=0.22 \xi^{2}$

then the $H=1$ surface, in spite of the fact that both surfaces have the same rms roughness.

\section{Percolation of the Contact Area}

The contact area between two solids depends on the magnification. When the magnification increases new surface roughness is observed and the (projected) contact area decreases. If the contact area percolate at the highest magnification, where all the surface roughness is included in the analysis, then no fluid leakage is possible.

The contact area depends on the rms-slope $\xi$ of the rough surface and on the effective elastic modulus $E^{*}=E /\left(1-v^{2}\right)$ of the rubber. Figure 4 shows the calculated area of real contact as a function of the nominal contact pressure divided by $E^{*}$ and by the rms-slope $\xi$ of the rough surface. In the calculation we have assumed a randomly rough surface and in this case the contact area only depends on the parameter $p /\left(E^{*} \xi\right)$ and takes the universal form shown in the figure. The contact area percolate when $p \approx 0.23 \xi E^{*}$.

Here we are interested in a cylinder rubber rib (radius $R$ ) squeezed against a flat hard surface with random roughness. From Hertz theory for cylinder geometry the force per unit length $F / L$ can be related to the effective modulus $E^{*}$ and the penetration $\delta$ (see inset in Fig. 5) as [10]:

$\frac{F}{L}=\frac{\pi}{4} E^{*} \delta$

The width of the contact region

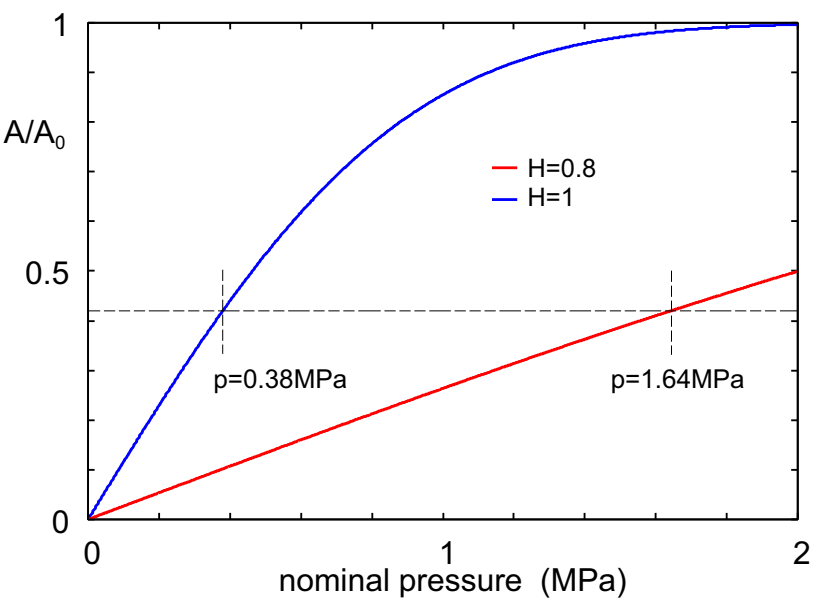

Fig. 6 The calculated area of real contact as a function of the nominal contact pressure for the $H=1$ (blue curve) and the $H=0.8$ (red) surfaces. For $E=10 \mathrm{MPa}$ and $v=0.5$ and using the power spectra shown in Fig. 2

$w=2(R \delta)^{1 / 2}$

The maximum contact pressure

$p_{0}=\left(\frac{E^{*} F / L}{\pi R}\right)^{1 / 2}$

From (6) and (8) we get

$\frac{\delta}{R}=\left(\frac{2 p_{0}}{E^{*}}\right)^{2}$

In order for the contact area to percolate in the high pressure region we need $p_{0}=0.23 \xi E^{*}$ (or larger) (see Fig. 4). Using this pressure in (9) we get

$\frac{\delta}{R}=0.22 \xi^{2}$

Thus in order for the seal not to leak the compression $\delta / R$ must be at least $0.22 \xi^{2}$, where $\xi$ is the rms-slope including all the roughness. In a typical case $\xi \approx 1$ and the theory predict that the compression must be at least $20 \%$ in order for the sealing to be completely tight. Note that the condition (10) is independent of the elastic modulus of the sealing material. The reason for this is that increasing the elastic modulus increases the contact pressure but at the same time the pressure needed to squeeze the rubber into the roughness profile gets higher, and the two effects cancel each other so the contact area (and the distribution of interfacial separation) remain unchanged. For real rubber materials, with non-linear stress-strain relations, this hold strictly true only if the microscopic strain in the asperity contact regions is similar to the macroscopic strain in the Hertz contact region. 
(a) no fluid pressure

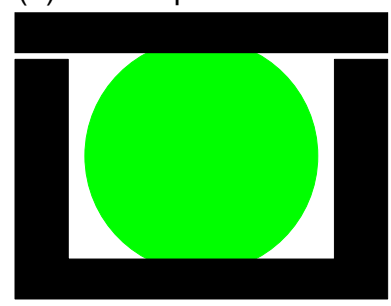

(b) with fluid pressure

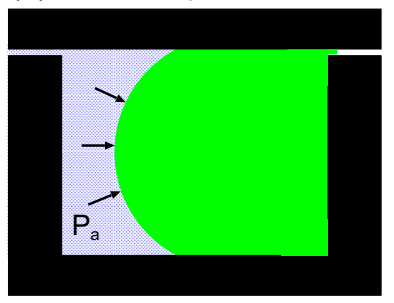

Fig. 7 a A rubber O-ring at fixed compression without fluid pressure. b Fluid pressure push the rubber O-ring against one of the confining walls

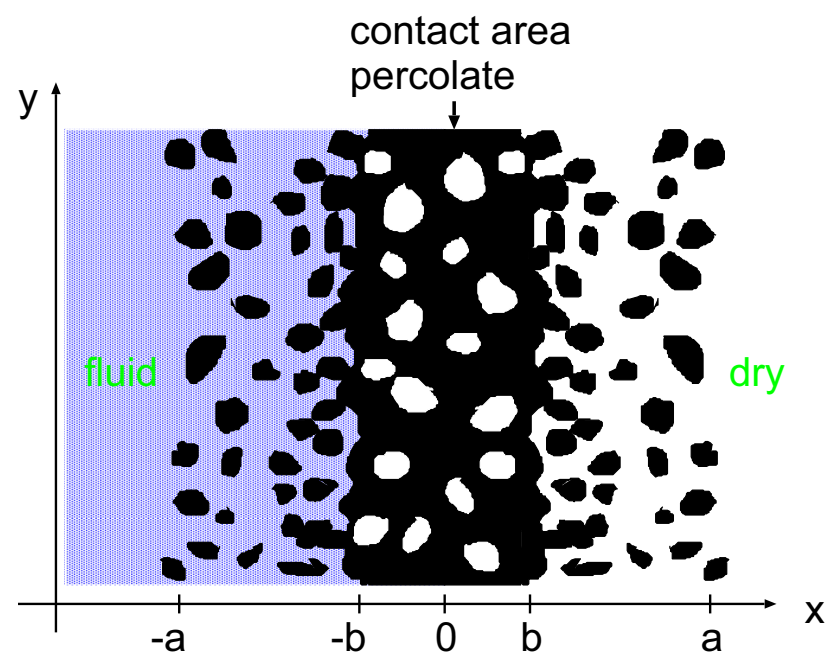

Fig. 8 The contact area (black) percolate in a strip $-b<x<b$ at the center of the Hertz line contact region. In this case no fluid (blue) can flow from the left to the right. (Schematic) (Color figure online)

Figure 5 shows the calculated area of real contact as a function of the penetration $\delta$ divided by the radius $R$ of the rubber cylinder and the square of the rms-slope $\xi$. The results is obtained from Fig. 4 using the relation (9) between the penetration and the pressure. The contact area percolate when the compression $\delta / R=0.22 \xi^{2}$.

Figure 6 shows the calculated area of real contact as a function of the nominal contact pressure for the $H=1$ (blue curve) and the $H=0.8$ (red) surfaces. Here we have used $E=10 \mathrm{MPa}$ and $v=0.5$ and the power spectra shown in Fig. 2. The contact area percolate when $p=0.38 \mathrm{MPa}$ for $H=1$ and for $p=1.64 \mathrm{MPa}$ for $H=0.8$.

\section{Fluid Leakage Theory and Dependency of the Leakrate on the Fluid Pressure}

The discussion in Sect. 3 is valid as long as the fluid pressure $p_{\mathrm{a}}$ on the high pressure side is much smaller than the maximum contact pressure. When this is not the case the fluid will increase the separation between the surfaces and result in an increased leakage rate. In particular, for large enough fluid pressure lift-off occur resulting in a catastrophic failure of the seal. In this section we will discuss the influence of the fluid pressure on the leakage rate.

The fluid pressure has two effects on a seal: it will elastically deform the seal and change its macroscopic shape (see Fig. 7) which will affect the contact pressure distribution. In addition, the fluid pressure will affect the surface separation in the nominal contact region. The first effect can be studied using standard Finite Element Method (FEM) calculations. In some applications, like in most O-ring applications (see Fig. 7), the nominal contact pressure increases nearly proportional to the fluid pressure while the fluid pressure at the contacting interface increases slower (it decreases from $p_{\mathrm{a}}$ at the inlet to $p_{\mathrm{b}}$ on the exit side and is hence smaller than $p_{\mathrm{a}}$ in the sealing region where the contact pressure is highest). Hence an O-ring seal may leak when the fluid pressure $p_{\mathrm{a}}$ is small while it is tight when the fluid pressure is high. This effect was observed recently in a syringe application (with a steel plunger road with a rubber O-ring seal) [14]. However, in some other sealing configurations the fluid pressure may have a negligible influence on the nominal squeezing pressure, and in these cases lift-off occur if the fluid pressure is high enough, as observed in Ref. [6].

Since the effect of the fluid pressure on the nominal contact pressure is well understood, and in order to have a clean situation, we will not considered this effect here, but we assume that the contact pressure distribution $p_{0}(x)$ is known and of Hertz-like form for simplicity.

We will study the influence of the fluid pressure on the leakrate for two cases, where, in the absence of the fluid, the contact area does not percolate or does percolate. In the former case the nominal contact pressure is below the percolation pressure everywhere in the nominal contact region, while in the second case the contact area percolate in a rectangular strip $-b<x<b$ at the center of the contact area (see Fig. 8).

\subsection{Theory}

We will calculate the fluid leakage using the effective medium theory developed in Ref. [6] (see also Ref. [15-20]). The ensemble averaged fluid flow current

$\mathbf{J}=-\sigma_{\text {eff }} \nabla p_{\text {fluid }}$ 
where $p_{\text {fluid }}$ is the ensemble averaged fluid pressure. The effective conductivity $\sigma_{\text {eff }}$ is calculated using the Bruggeman effective medium theory. In this theory enters the probability distribution of interfacial separations which is calculated using the Persson contact mechanics theory. For the cylinder geometry which interest us here the fluid pressure $p_{\text {fluid }}(x)$ and the (asperity) contact pressure (also ensemble averaged) $p_{\text {cont }}(x)$ depends only on the coordinate $x$ orthogonal to the cylinder axis. If we denote

$S(x)=\int_{0}^{x} d x^{\prime} \sigma_{\text {eff }}^{-1}\left(p_{\text {cont }}\left(x^{\prime}\right)\right)$

then the leakrate (volume per unit time) is given by

$\dot{Q}=\frac{L_{y}\left(p_{\mathrm{a}}-p_{\mathrm{b}}\right)}{S\left(L_{x}\right)}$

where $L_{x}=2 a$ is the width of the nominal contact region in the fluid flow direction, orthogonal to the cylinder axis. The fluid pressure

$p_{\text {fluid }}(x)=p_{\mathrm{a}}-\left(p_{\mathrm{a}}-p_{\mathrm{b}}\right) \frac{S(x)}{S\left(L_{x}\right)}$

The (ensemble averaged) pressure acting on the rubber surface is

$p_{0}(x)=p_{\text {cont }}(x)+p_{\text {fluid }}(x)$

In the absence of the fluid pressure the contact pressure is assumed to be Hertz-like:

$p_{0}(x)=p_{0}\left(1-\left(\frac{x}{a}\right)^{2}\right)^{1 / 2}$

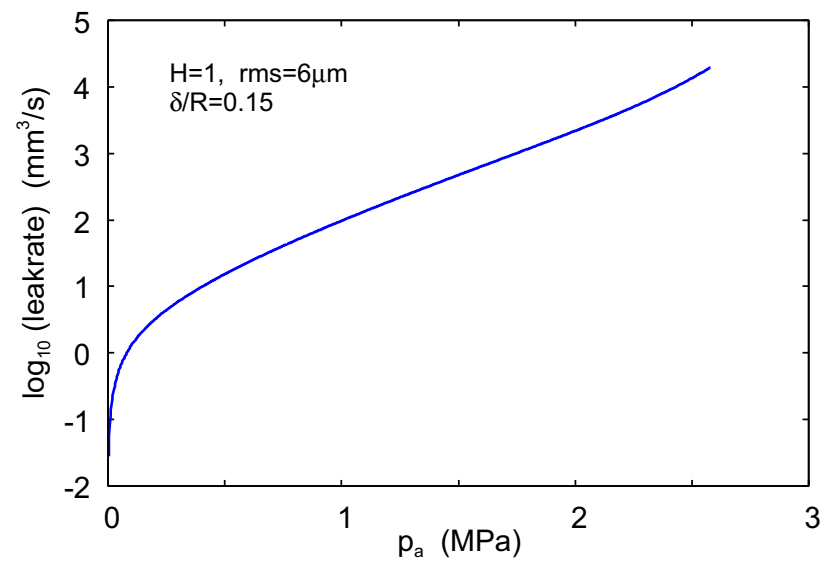

Fig. 9 The logarithm of the leakrate as a function of the fluid pressure $p_{\mathrm{a}}$ for the $H=1$ and $h_{\mathrm{rms}}=6 \mu \mathrm{m}$ and for the compression $\delta / R=0.15$. The seal is $L_{y}=1 \mathrm{~m}$ long. The contact area does not percolate for any fluid pressure

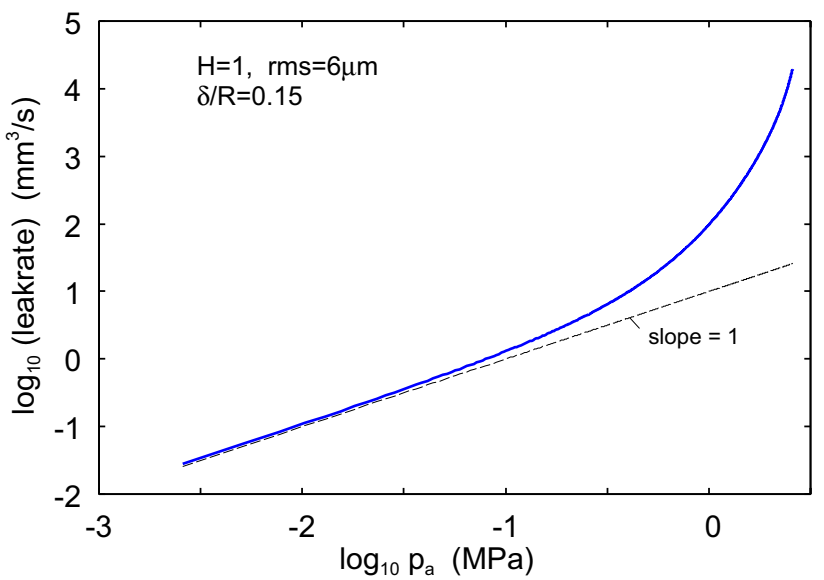

Fig. 10 The same as in Fig. 9 but with the pressure scale logarithmic. The strait line has the slope 1 showing that for fluid pressures $p_{\mathrm{a}}<0.1 \mathrm{MPa}$ the leakrate is proportional to the fluid pressure

This pressure result from the macroscopic deformations of the rubber. Thus a small change in the interfacial surface separation induced by the fluid pressure will have a negligible influence on the (ensemble averaged) pressure acting on the rubber surface except in the entrance region $(x \approx-a)$ where $p_{0}(x)$ is very small $\left[p_{0}(x) \rightarrow 0\right.$ as $\left.x \rightarrow-a\right]$. In this region lift-off will occur and the pressure acting on the rubber will equal the fluid pressure $p_{\mathrm{a}}$. Thus we take $p_{0}(x)$ equal to $p_{\mathrm{a}}$ in the lift-off region and equal to (16) in the remaining part of the contact region.

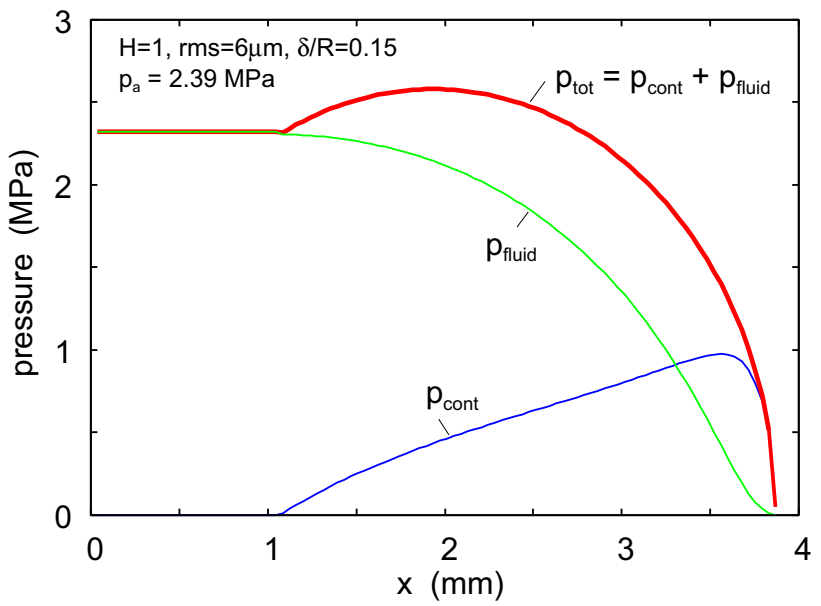

Fig. 11 The dependency of the fluid pressure $p_{\text {fluid }}$ (green line), the contact pressure $p_{\text {cont }}$ (blue line) and the total pressure $p_{\text {tot }}=p_{\text {cont }}+p_{\text {fluid }}$ (red line) on the coordinate $x$ in the nominal rubber-countersurface contact area. The contact pressure $p_{\text {cont }}$ is below the percolation pressure $p_{\text {perc }} \approx 7.6 \mathrm{MPa}$ everywhere. For $H=1$, $\delta / R=0.15$ and $h_{\mathrm{rms}}=6 \mu \mathrm{m}$ and the fluid pressure on the high pressure side $p_{\mathrm{a}}=2.39 \mathrm{MPa}$ (Color figure online) 


\subsection{Contact Area Does not Percolate}

In this case open fluid flow channels occur at the interface for all applied fluid pressures $p_{\mathrm{a}}$. As an illustration consider the $H=1$ surface but with a roughness scaled up with a factor of 20 so that $h_{\text {rms }}=6 \mu \mathrm{m}$. For this case the rms-slope will be 20 times bigger than for the $H=1$ surface with $h_{\mathrm{rms}}=0.3 \mu \mathrm{m}$. Thus $\xi \approx 20 \times 0.103 \approx 2$ and the contact area at the center of the contact region will percolate for the compression $\delta / R>0.22 \xi^{2} \approx 0.88$. Hence, for the compression 0.15 and 0.3 the contact area will not percolate and the seal will leak already for arbitrary small fluid pressures $p_{\mathrm{a}}$. To illustrate this case Fig. 9 shows the logarithm of the leakrate as a function of the fluid pressure $p_{\mathrm{a}}$ for the compression $\delta / R=0.15$. Figure 10 shows the same results but now as a function of the logarithm of the fluid pressure $p_{\mathrm{a}}$. The strait line has the slope 1 showing that for fluid pressures $p_{\mathrm{a}}<0.1 \mathrm{MPa}$ the leakrate is proportional to the fluid pressure as expected when the separation between the surfaces is unchanged $[15,16]$.

Figure 11 shows the dependency of the fluid pressure $p_{\text {fluid }}$ (green line), the contact pressure $p_{\text {cont }}$ (blue line) and the total pressure $p_{\text {tot }}=p_{\text {cont }}+p_{\text {fluid }}$ (red line) on the coordinate $x$ in the nominal rubber-countersurface contact area. The fluid pressure on the high pressure side $p_{\mathrm{a}}=2.39 \mathrm{MPa}$. Note that the fluid pressure has separated the surfaces on the

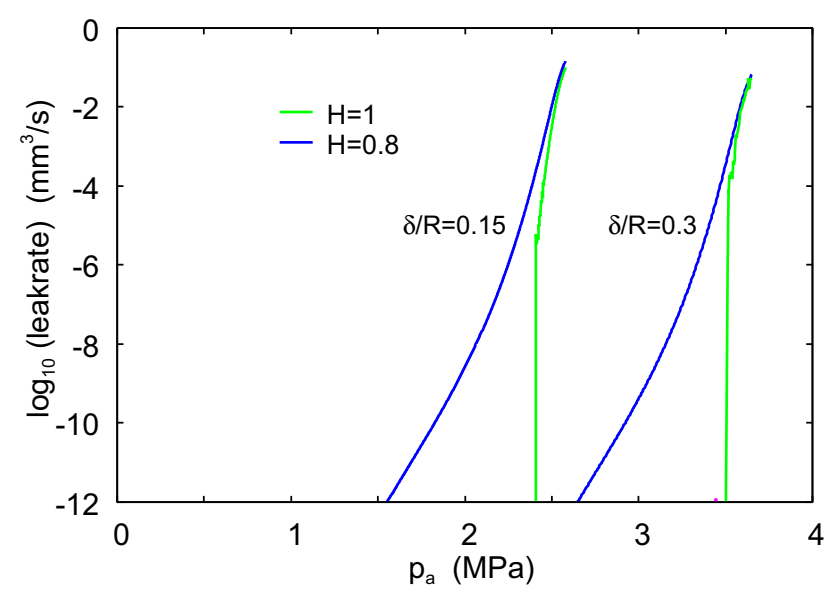

Fig. 12 The logarithm of the leakrate as a function of the fluid pressure $p_{\mathrm{a}}$ for the $H=1$ (green lines) and $H=0.8$ (blue lines). The seal is $L_{y}=1 \mathrm{~m}$ long. The two curves at the lowest pressure are for the compression $\delta / R=0.15$ and the two upper ones for $\delta / R=0.3$. For low water pressure the contact area percolate and the leakage is negligible. Higher compression result in higher contact pressure and a higher water pressure is needed to separate the surfaces enough in order for the contact area not to percolate. For large enough water pressure the surface separation becomes so large that the surface roughness has negligible influence on the leakage rate. This is the reason the blue ( $H=0.8$ surface) and green ( $H=1$ surface) lines for each compression merge at the highest water pressures (Color figure online) high pressure side so that no real contact occur between the rubber and the countersurface for $0<x<1 \mathrm{~mm}$.

\subsection{Contact Area Percolate}

We consider now the case where, when the fluid pressure vanish, the contact area percolate in a strip at the center of the contact. The percolated area will act as a barrier towards fluid leakage (see Fig. 8). In this case it is necessary to discuss how the seal was mounted. If the seal was mounted in the dry state regions with compressed air may form in the area where the contact area percolate. If the seal was mounted in the fluid, fluid filled (and pressurized) regions (lakes) may form in the percolated area. In order to have a well-defined system in this case we will assume that there is a very small fluid leak current through the contact region where the contact area percolate. This may be due to diffusion of water molecules through the rubber matrix. In this case, after a long enough contact time, a well-defined stationary state will be formed, where some (extremely small) leakage occur even when the contact area percolate. We will describe this very small fluid flow by a constant (extremely small) flow conductivity $\sigma_{\text {eff }}^{*}$ in the region where the contact area percolate. The results presented below does not depend on the magnitude of $\sigma_{\text {eff }}^{*}$ as long as it is extremely small.

To illustrate the case when the contact area percolate for small fluid pressures we consider the two systems studied in Sect. 3 where $\xi=0.103$ (surface $H=1$ ) and $\xi=0.445$ (surface $H=0.8$ ). For these two cases the contact area percolate for the compression $\delta / R \approx 0.22 \xi^{2}=0.0023$ and 0.0436 , respectively.

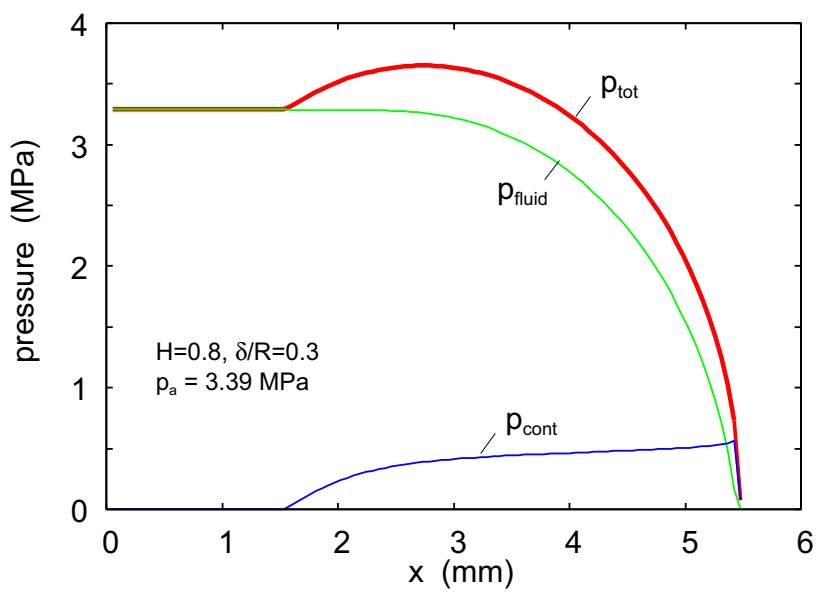

Fig. 13 The dependency of the fluid pressure $p_{\text {fluid }}$ (green line), $p_{\text {cont }}$ (blue line) and the total pressure $p_{\text {tot }}=p_{\text {cont }}+p_{\text {fluid }}$ (red line) on the coordinate $x$ in the nominal rubber-countersurface contact area. The fluid pressure is so high that the contact pressure $p_{\text {cont }}$ is always below the percolation pressure $p_{\text {perc }} \approx 1.64 \mathrm{MPa}$ (see Fig. 6). For $H=0.8, \delta / R=0.3$ and the fluid pressure on the high pressure side $p_{\mathrm{a}}=3.39 \mathrm{MPa}$ (Color figure online) 


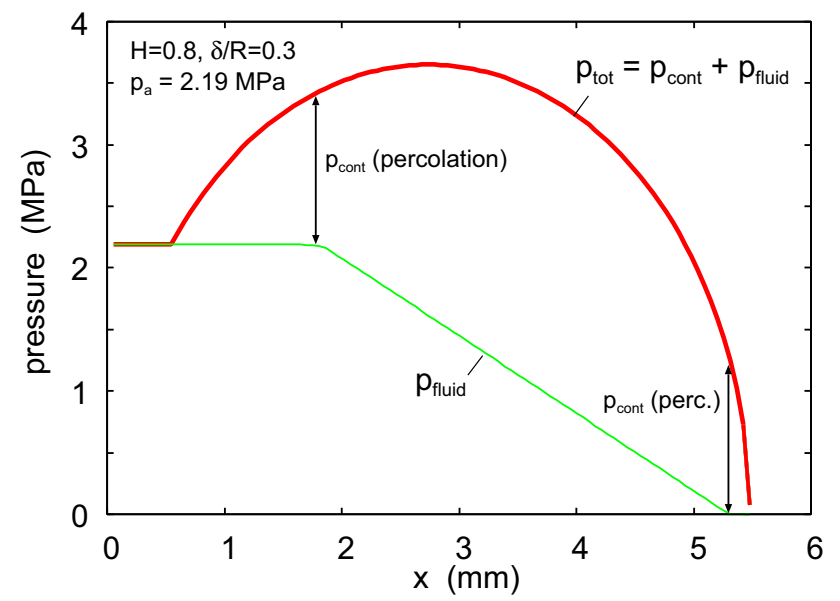

Fig. 14 The dependency of the fluid pressure $p_{\text {fluid }}$ (green line) and the total pressure $p_{\text {tot }}=p_{\text {cont }}+p_{\text {fluid }}$ (red line) on the coordinate $x$ in the nominal rubber-countersurface contact area. The contact pressure $p_{\text {tot }}-p_{\text {fluid }}$ is above the percolation pressure $p_{\text {perc }}$ in a region $1.80 \mathrm{~mm}<x<5.25 \mathrm{~mm}$. In this region the fluid pressure is decreasing linearly with $x$ as a result of assuming a contact fluid conductivity in this region (see text). For $H=0.8, \delta / R=0.3$ and the fluid pressure on the high pressure side $p_{\mathrm{a}}=2.19 \mathrm{MPa}$ (Color figure online)

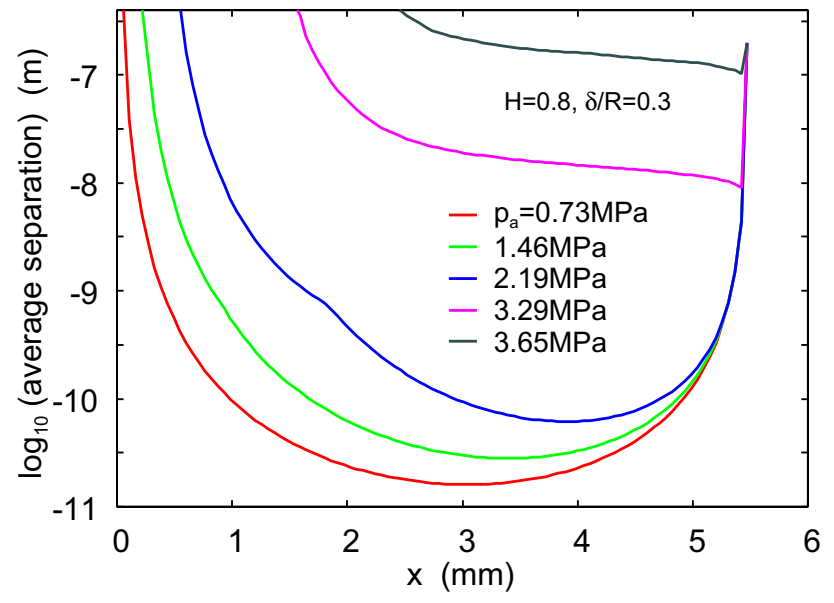

Fig. 15 The logarithm of the average surface separation as a function the coordinate $x$ in the nominal rubber-countersurface contact area. We show results for several fluid pressures indicated in the figure $\left(p_{\mathrm{a}}=0.73,1.46,2.19,3.29\right.$ and $\left.3.65 \mathrm{MPa}\right)$

Figure 12 shows the logarithm of the leakrate as a function of the fluid pressure $p_{\mathrm{a}}$ for the $H=1$ (green lines) and $H=0.8$ (blue lines) surfaces. The two curves at the lowest pressure are for the compression $\delta / R=0.15$, and the two upper ones for $\delta / R=0.3$. For low water pressure the contact area percolate and the leakage is negligible. Higher compression result in higher contact pressure and a higher water pressure is needed to separate the surfaces enough in order for the contact area not to percolate. For large enough water pressure the surface separation becomes so large that only the longest wavelength roughness (with the largest amplitude) matter. This is the reason the blue ( $H=0.8$ surface) and green ( $H=1$ surface) lines for each compression merge at the highest water pressures. At even higher water pressure the surface roughness has a negligible influence on the leakage rate.

Figure 13 shows the dependency of the fluid pressure $p_{\text {fluid }}$ (green line), the contact pressure $p_{\text {cont }}$ (blue line), and the total pressure $p_{\text {tot }}=p_{\text {cont }}+p_{\text {fluid }}$ (red line) on the coordinate $x$ in the nominal rubber-countersurface contact area for $H=0.8$ and $\delta / R=0.3$. The fluid pressure $p_{\mathrm{a}}=3.39 \mathrm{MPa}$ is so high that the contact pressure $p_{\text {cont }}$ is always below the percolation pressure $p_{\text {perc }}=1.64 \mathrm{MPa}$. For the same system, in Fig. 14 we show the dependency of the fluid pressure and the total pressure on the coordinate $x$ when the fluid pressure on the high pressure side $p_{\mathrm{a}}=2.19 \mathrm{MPa}$. In this case the contact pressure $p_{\text {cont }}=p_{\text {tot }}-p_{\text {fluid }}$ is above the percolation pressure $p_{\text {perc }}$ in a region $1.80 \mathrm{~mm}<x<5.25 \mathrm{~mm}$. In this region the fluid pressure is decreasing linearly with $x$ as a result of the assumption of a constant fluid conductivity in this region. The result in Fig. 14 is of no practical importance since the leakage rate is negligible small (and determined by $\sigma_{\text {eff }}^{*}$ ) but the figure shows how deep into the contact the fluid penetrate by lift-off (to $x \approx 0.5 \mathrm{~mm}$ ) and by infiltration into the net-yet percolated contact region (to $x \approx 1.8 \mathrm{~mm}$ ). Note also that the fluid pressure vanish in a narrow $x$-region (from $x \approx 5.25$ to $5.4 \mathrm{~mm}$ ) at the exit of the contact region, where the contact area is not percolated. Thus in this $x$-region the resistance to fluid flow is negligible because of the extremly small leak correct which result from the finite but extremly small flow conductivity $\sigma_{\text {eff }}^{*}$ in the percolated region.

Figure 15 shows the logarithm of the average surface separation as a function the coordinate $x$ in the nominal rubbercountersurface contact area. We show results for the fluid pressures $p_{\mathrm{a}}=0.73,1.46,2.19,3.29$ and $3.65 \mathrm{MPa}$.

\section{Discussion}

In the study above we have assumed linear elastic materials. However, rubber materials are highly non-linear with a stress-strain curve $\sigma(\epsilon)$ which, for rubber with filler particles, first exhibiting strain softening followed by strain stiffening. The simplest way to approximately include this effect in the calculations presented above is to use an effective modulus $E_{\text {eff }}(\epsilon)=\sigma(\epsilon) / \epsilon$, where the strain $\epsilon$ is the typical strain in the asperity contact regions. The macroscopic contact pressure profile $p_{0}(x)$ can be calculated using the FEM approach including the full non-linear modulus e.g., using the Mooney-Rivlin material description [21]. The time (or frequency) dependency of the modulus can also be included 
in an approximate way as a time dependent factor determined by DMA measurements.

\section{Summary and Conclusion}

A seal will not leak if the area of real contact percolate. In design manuals for rubber seals an upper limit for the rms roughness (or the arithmetical mean deviation) $h_{\mathrm{rms}}$ is often given. However, $h_{\mathrm{rms}}$ is determined by the most longwavelength roughness which usually has a negligible influence on the area of real contact. For non-adhesive contact between solids with randomly rough surfaces the area of real contact depends only on the rms-slope of the combined roughness. The rms-slope depends on all surface roughness wavelength components, and in particular on the short wavelength roughness.

Static rubber seals are usually confined between the surfaces of two (nearly) rigid solids at a fixed compression $\delta / R$ (see Fig. 1). I have show that if $\delta / R$ is fixed and if the fluid pressure is negligible compared to the rubber-countersurface pressure, then the fluid leakage rate does not depend on the elastic modulus of the rubber seal.

We have shown that in order for the seal not to leak the compression $\delta / R$ must be at least $0.22 \xi^{2}$, where $\xi$ is the rmsslope including all the roughness. In a typical case $\xi \approx 1$ and the theory predict that the compression must be at least $20 \%$ in order for the sealing to be completely tight. Note that the condition $\delta / R \approx 0.22 \xi^{2}$ is independent of the elastic modulus of the sealing material. The reason for this is that increasing the elastic modulus increases the contact pressure but at the same time the pressure needed to squeeze the rubber into the roughness profile gets higher, and the two effects cancel each other so the contact area (and the distribution of interfacial separation) remain unchanged. For real rubber materials, with non-linear stress-strain relations, this hold strictly true only if the microscopic strain in the asperity contact regions is similar to the macroscopic strain in the Hertz contact region.

We have discussed the influence of the fluid pressure on the interfacial separation and the leakage of seals. When the fluid pressure increase towards the (maximum) contact pressure lift-off occur resulting in catastrophic increase in the leakage. In some applications, like in most O-ring applications, the contact pressure increases nearly proportional to the fluid pressure and in these applications no lift-off is expected for any fluid pressure but the seal may anyway fail for very high fluid pressures due to high tensile stresses acting on the rubber as it is squeezed into the gap between the shaft and the housing.
Funding Open Access funding enabled and organized by Projekt DEAL. The authors have not disclosed any funding.

\section{Declarations}

Conflict of interest The author has no financial or non-financial interests to disclose.

Open Access This article is licensed under a Creative Commons Attribution 4.0 International License, which permits use, sharing, adaptation, distribution and reproduction in any medium or format, as long as you give appropriate credit to the original author(s) and the source, provide a link to the Creative Commons licence, and indicate if changes were made. The images or other third party material in this article are included in the article's Creative Commons licence, unless indicated otherwise in a credit line to the material. If material is not included in the article's Creative Commons licence and your intended use is not permitted by statutory regulation or exceeds the permitted use, you will need to obtain permission directly from the copyright holder. To view a copy of this licence, visit http://creativecommons.org/licenses/by/4.0/.

\section{References}

1. Tiwari, A., Persson, B.N.J.: Cylinder-flat contact mechanics with surface roughness. Tribol. Lett. 69, 4 (2021)

2. O-rings, Trelleborg sealing solutions, https://www.peter2000.co. uk/aviation/tb20-experience/trelleborg-o_ring_gb.pdf

3. How Surface Finish Affects Sealing Performance, Eclipse Engineering Inc., https://eclipseseal.com/blog/seals/surface-finishaffects-sealing-performance/

4. Persson, B.N.J., Albohr, O., Tartaglino, U., Volokitin, A.I., Tosatti, E.: On the nature of surface roughness with application to contact mechanics, sealing, rubber friction and adhesion. J. Phys.: Condens. Matter 17, R1 (2005)

5. Dapp, W.B., Lücke, A., Persson, B.N.J., Müser, M.H.: Self-affine elastic contacts: percolation and leakage. Phys. Rev. Lett. 108, 244301 (2012)

6. Lorenz, B., Persson, B.N.J.: Time-dependent fluid squeeze-out between solids with rough surfaces. Eur. Phys. J. E 32, 281 (2010)

7. Barabasi, A..-L., Stanley, H..E.: Fractal Concepts in Surface Growth. Cambridge University Press, Cambridge (1995)

8. Persson, B.N.J.: On the fractal dimension of rough surfaces. Tribol. Lett. 54, 99 (2014)

9. Jacobs, T.D.B., Junge, T., Pastewka, L.: Quantitative characterization of surface topography using spectral analysis. Surf. Topogr.: Metrol. Prop. 5, 013001 (2017)

10. Johnson, K.. L.: Contact Mechanics. Cambridge University Press, Cambridge (1985)

11. Persson, B.N.J.: Theory of rubber friction and contact mechanics. J. Chem. Phys. 115, 3840 (2001)

12. Afferrante, L., Bottiglione, F., Putignano, C., Persson, B.N.J., Carbone, G.: Elastic contact mechanics of randomly rough surfaces: an assessment of advanced asperity models and Persson theory. Tribol. Lett. 66, 1 (2018)

13. Almqvist, A., Campana, C., Prodanov, N., Persson, B.N.J.: Interfacial separation between elastic solids with randomly rough surfaces: comparison between theory and numerical techniques. J. Mech. Phys. Solids 59, 2355 (2012)

14. Huon, C., Tiwari, A., Rotella, C., Mangiagalli, P., Persson, B.N.J.: Air, helium and water leakage in rubber O-ring seals with application to syringes. Tribol. Lett. (2021). https://doi.org/10.21203/rs.3. rs-979477/v1 
15. Lorenz, B., Persson, B.N.J.: Leak rate of seals: effective-medium theory and comparison with experiment. Eur. Phys. J. E 31, 159 (2010)

16. Lorenz, B., Persson, B.N.J.: Leak rate of seals: comparison of theory with experiment. EPL (Europhys. Lett.) 86, 44006 (2009)

17. Persson, B.N.J.: Comments on the theory of fluid flow between solids with anisotropic roughness. Tribol. Lett. 69, 2 (2021)

18. Wang, A., Müser, M.H.: Percolation and Reynolds flow in elastic contacts of isotropic and anisotropic, randomly rough surfaces. Tribol. Lett. 69, 1 (2021)

19. Scaraggi, M.: The friction of sliding wet textured surfaces: the Bruggeman effective medium approach revisited. Proc. R. Soc. A: Math. Phys. Eng. Sci. 471, 20140739 (2015)
20. Bottiglione, F., Carbone, G., Mangialardi, L., Mantriota, G.: Leakage mechanism in flat seals. J. Appl. Phys. 106, 104902 (2009)

21. Mihai, L.A., Goriely, A.: How to characterize a nonlinear elastic material? A review on nonlinear constitutive parameters in isotropic finite elasticity. Proc. R. Soc. A 473, 20170607 (2017)

Publisher's Note Springer Nature remains neutral with regard to jurisdictional claims in published maps and institutional affiliations. 